\title{
Nonlinear dynamics of a microswimmer in Poiseuille flow
}

\author{
Andreas Zöttl and Holger Stark \\ Institut fur Theoretische Physik, Technische Universität Berlin, Hardenbergstrasse 36, 10623 Berlin, Germany
}

(Dated: September 21, 2018)

\begin{abstract}
We study the three-dimensional dynamics of a spherical microswimmer in cylindrical Poiseuille flow which can be mapped onto a Hamiltonian system. Swinging and tumbling trajectories are identified. In 2D they are equivalent to oscillating and circling solutions of a mathematical pendulum. Hydrodynamic interactions between the swimmer and confining channel walls lead to dissipative dynamics and result in stable trajectories, different for pullers and pushers. We demonstrate this behavior in the dipole approximation of the swimmer and with simulations using the method of multi-particle collision dynamics.
\end{abstract}

PACS numbers: 47.63.Gd, 47.63.mf, 47.61.-k

Microswimmers often have to respond to fluid flow and confining boundaries, like sperm cells in the Fallopian tubes [1] or pathogens in blood vessels [2]. Artificial microswimmers constructed with the vision to act as drugdeliverers in the human body [3] would have to swim in narrow channels like arteries. Two properties influence the swimming in microchannels. On the one hand, vortices in flow reorient the swimming direction of microorganisms. In simple shear flow, for example, microswimmers tumble due to a constant flow vorticity [4]. Vortices in Poiseuille flow in combination with bottom-heaviness due to gravitation lead to stable orientations of swimming algae cells [5]. On the other hand, microorganisms swimming near surfaces are trapped by hydrodynamic interactions [6] and ultimately escape with the help of rotational diffusion [10]. Finally, bacteria in Poiseuille flow show a net-upstream flux at the walls due to the interplay of confinement and flow vorticity [7, 8]. All these examples show there is genuine interest in understanding generic features of microorganisms and artificial swimmers in Poiseuille flow.

In this letter we demonstrate that the dynamics of a simple spherical microswimmer in a cylindrical Poiseuille flow can be mapped onto a conservative dynamical system with the Hamiltonian as a constant of motion. In analogy to the oscillating and circling solutions of a mathematical pendulum, we discuss in detail the swinging and tumbling motion of the microswimmer in 2D and generalize them to three dimensions. Hydrodynamic interactions with the channel wall treated in the dipole approximation introduce dissipation and the microswimmer assumes specific stable swimming trajectories depending on its type as puller or pusher.

We first introduce the geometry. We consider a pointlike microswimmer that moves with a constant intrinsic swimming speed $v_{0}$ in a cylindrical microchannel where a Poiseuille flow is imposed. Using a cylindrical coordinate system $(\rho, \varphi, z)$ with the coordinate basis $(\hat{\boldsymbol{\rho}}, \hat{\boldsymbol{\varphi}}, \hat{\mathbf{z}})$, the flow is given by $\mathbf{v}_{f}=v_{f}\left(1-\rho^{2} / R_{\mathrm{Ch}}^{2}\right) \hat{\mathbf{z}}$, where $v_{f}$ is the maximum flow speed in the center of the channel [Fig. 1(a)]. In the absence of noise the equations of mo- (a)
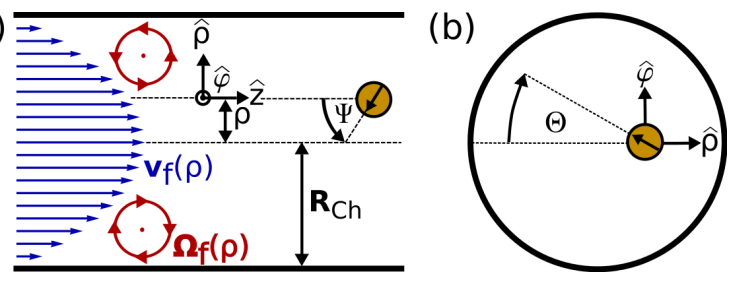

Figure 1. Swimmer in Poiseuille flow. (a) Flow profile $\mathbf{v}_{f}(\rho)$, cylindrical coordinate system with coordinate basis $(\hat{\boldsymbol{\rho}}, \hat{\boldsymbol{\varphi}}, \hat{\mathbf{z}})$ and orientation angle $\Psi$ for the projected orientation into the $\rho$ - $z$-plane. When $e_{\varphi}=\sin \Theta=0$, the motion is two-dimensional. Note that the sign of vorticity $\boldsymbol{\Omega}_{f}$ changes when crossing the centerline. (b) Cross section of the microchannel. The orientation in $\varphi$-direction defines the angle $\Theta$.

tion for the swimmer position $\mathbf{r}$ and orientation $\hat{\mathbf{e}}$ are given by

$$
\frac{d}{d t} \mathbf{r}=v_{0} \hat{\mathbf{e}}+\mathbf{v}_{f}, \quad \frac{d}{d t} \hat{\mathbf{e}}=\frac{1}{2} \boldsymbol{\Omega}_{f} \times \hat{\mathbf{e}}
$$

where $\boldsymbol{\Omega}_{f}=\nabla \times \mathbf{v}_{f}=v_{f} \rho / R_{\mathrm{Ch}}^{2} \hat{\boldsymbol{\varphi}}$ is the flow vorticity. The swimmer orientation $\hat{\mathbf{e}}=e_{\rho} \hat{\boldsymbol{\rho}}+e_{\varphi} \hat{\boldsymbol{\varphi}}+e_{z} \hat{\mathbf{z}}$ has the components

$$
e_{\rho}=-\cos \Theta \sin \Psi, e_{\varphi}=\sin \Theta, e_{z}=-\cos \Theta \cos \Psi,
$$

where $\Psi \in\{-\pi, \pi\}$ is the angle in the $\rho$ - $z$-plane [Fig. [1(a)] and $\Theta \in\{-\pi / 2, \pi / 2\}$ measures the orientation in the azimuthal $\varphi$-direction [Fig. 1(b)]. We note that $|\Psi|<\pi / 2$ means upstream and $|\Psi|>\pi / 2$ downstream orientation, respectively. In the following we use rescaled units, $\rho / R_{\mathrm{Ch}} \rightarrow \rho \in\{0,1\}, z / R_{\mathrm{Ch}} \rightarrow z$ and $t / t_{0} \rightarrow t$ with $t_{0}=R_{\mathrm{Ch}} / v_{0}$. We also introduce the dimensionless flow speed $\bar{v}_{f}=v_{f} / v_{0}$, which is the only essential parameter in our problem.

First, we discuss 2D solutions of Eqs. (1) since they already capture many aspects of the swimmer dynamics. When $\Theta=0$, the trajectories of the swimmer are restricted to two dimensions, for example, to the $x-z$ plane, $x \in\{-1,1\}$. Due to the translational symmetry in $z$-direction, only the equations for $x$ and $\Psi$ are coupled, 
and Eqs. (1) give $\dot{x}=-\sin \Psi, \dot{\Psi}=\bar{v}_{f} x$. Eliminating $x$ results in

$$
\ddot{\Psi}=\bar{v}_{f} \sin \Psi
$$

which is the equation of motion of the mathematical pendulum. Since in this analogy $x$ plays the role of velocity, we can immediately write down the 2D Hamiltonian

$$
H_{2 \mathrm{D}}=\frac{1}{2} \bar{v}_{f} x^{2}+1-\cos \Psi
$$

as a conserved quantity. Fig. 2] shows the $x-\Psi$ phase space and typical trajectories $z(x)$ for several flow strengths $\bar{v}_{f}$. In analogy to the pendulum two swimming states exist. The flow vorticity rotates the upstream oriented microswimmer always towards the center. Hence, the swimmer performs a swinging motion around the centerline of the channel for $H_{2 \mathrm{D}}<2$ which corresponds to the oscillating solution of the pendulum [e. g. blue trajectory of Fig. 2(a)]. For small amplitudes $(\Psi \ll 1)$ the swinging frequency is $\omega_{0}=\sqrt{v_{f}}$. When the upstream oriented swimmer moves exactly in the center of the channel (stable fixed point), the Hamiltonian is zero. Downstream swimming along the centerline $(\Psi=\pi)$ is an unstable fixed point. After a slight disturbance of $x=0$, vorticity rotates the swimmer away from the centerline. The swimmer performs a tumbling motion $\left(H_{2 \mathrm{D}}>2\right)$ which corresponds to the circling solution of the pendulum [green trajectory of Fig. 2(c)]. At $H_{2 \mathrm{D}}=2$, the separatrix $x^{2}=2(\cos \Psi+1) / \bar{v}_{f}$ divides the swinging and tumbling region in phase space [red curves in the phase portraits of Fig. 2. Since the Poiseuille flow is bounded by the channel walls, tumbling motion only occurs for $\bar{v}_{f}>4$ [Fig. 2(c)]. Meaning sufficiently strong vorticity to prevent the swimmer from crossing the centerline.

If we only consider steric interactions of the swimmer with the channel wall, the swimmer crashes into the wall at $|x|=1$ for $H_{2 \mathrm{D}}>\bar{v}_{f} / 2$, reorients due to the flow vorticity towards the upstream orientation, and leaves the wall at $\Psi=0$ with $H_{2 \mathrm{D}}^{\max }=\bar{v}_{f} / 2$. The swimmer then performs a swinging motion between the walls with maximum amplitude $|x|=1$ for $\bar{v}_{f}<4$ [green trajectory in Fig. 2(a)]. So for $\bar{v}_{f}<4$ the swimmer always enters a swinging motion oriented upstream, at the latest after contact with the wall, whereas it tumbles close to the wall for $\bar{v}_{f}>4$.

To determine the full 2D trajectory in the microchannel, we solve the dynamic equation for $z(t)$,

$$
\dot{z}=\bar{v}_{f}\left[1-x(t)^{2}\right]-\cos \Psi(t) .
$$

A careful analysis reveals the following. The swimmer always moves upstream $(\dot{z}<0)$, when $\bar{v}_{f}<1-H_{2 \mathrm{D}}$, as shown in Fig. 2(a). When the flow is strong $\left(\bar{v}_{f}>1+2 H_{2 \mathrm{D}}\right)$, the swimmer always drifts downstream $(\dot{z}>0)$, while swinging or tumbling [Fig. 2(c)]. In between, mixed up- and downstream segments within one trajectory [Fig. 2(b)]
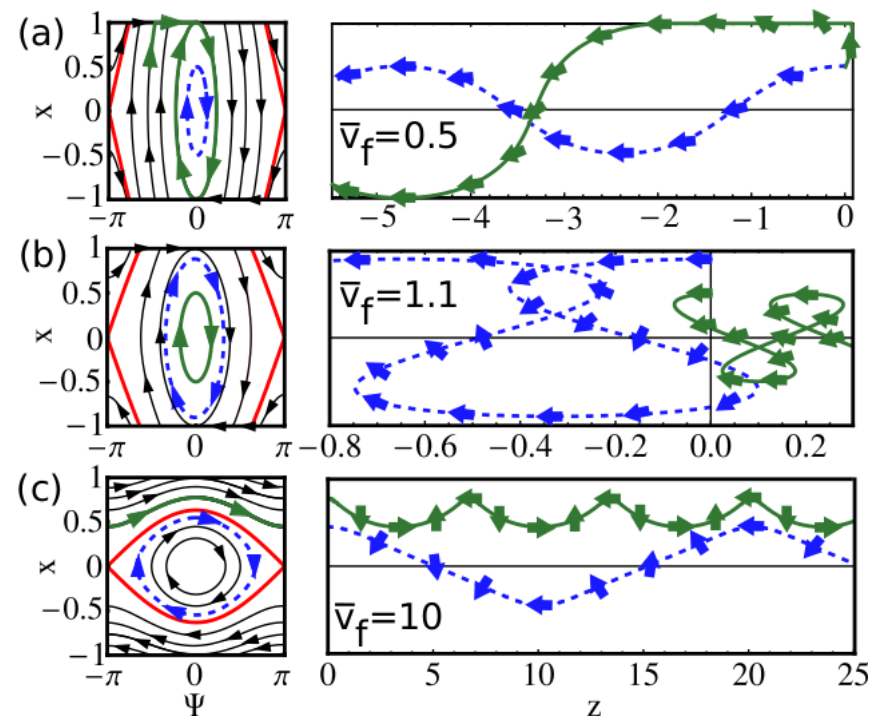

Figure 2. Phase spaces $x-\Psi$ (left) and typical trajectories $z(x)$ (right) for several flow strengths $\bar{v}_{f}$. All trajectories start at $z=0$. (a) upstream motion, (b) intermediate motion and (c) downstream motion. Note the various scales for the z-axis. The arrows indicate the orientation vector $\hat{\mathbf{e}}$ of the swimmer.

exist but a net upstream motion only occurs for $\bar{v}_{f} \lesssim$ $1+H_{2 \mathrm{D}} / 2$ [blue line in Fig. 2(b)].

For a non-zero azimuthal component, $e_{\varphi}=\sin \Theta \neq$ 0 , the swimmer trajectory is three-dimensional. Using Eqs. (11) and Eqs. (21), we obtain three coupled equations for $\Psi, \Theta$ and $\rho$,

$$
\begin{aligned}
\dot{\rho} & =-\cos \Theta \sin \Psi \\
\dot{\Psi} & =\bar{v}_{f} \rho-\sin \Theta \tan \Theta \cos \Psi / \rho \\
\dot{\Theta} & =\sin \Theta \sin \Psi / \rho .
\end{aligned}
$$

Due to translational symmetry in $z$-direction and rotational symmetry about the channel axis, Eqs. (6) do not depend on $z$ and $\varphi$. We are able to identify two constants of motion,

$$
\begin{aligned}
L_{z} & =\rho \sin \Theta \\
H & =\frac{1}{2} \bar{v}_{f} \rho^{2}+1-\cos \Psi \cos \Theta,
\end{aligned}
$$

where $L_{z}$ is proportional to the angular momentum of the swimmer in $z$-direction. Due to this constant the sign of $\Theta$ along a swimmer trajectory does not change. Eliminating $\Theta$ from Eqs. (6), reduces the equations of motions to $\frac{\partial H}{\partial \Psi}=-\dot{\rho}, \frac{\partial H}{\partial \rho}=\dot{\Psi}$. So, $H$ again plays the role of a Hamiltonian for the conjugate variables $\Psi$ and $\rho$.

The intersection of the two constants of motion gives the orbit of the swimmer in $\rho-\Psi-\Theta$ phase space [Fig. [3(a,b)]. The stable fixed points of Eqs. (6) lie on the fixed-point line $\left(\rho^{*}=\sqrt{\sin \Theta^{*} \tan \Theta^{*} / \bar{v}_{f}}, \Psi^{*}=0\right)$, drawn in Fig. 3(a). Swimming at a fixed point corresponds to a helical trajectory [Fig. 3 (c)]. The swimmer moves up- 
(a)

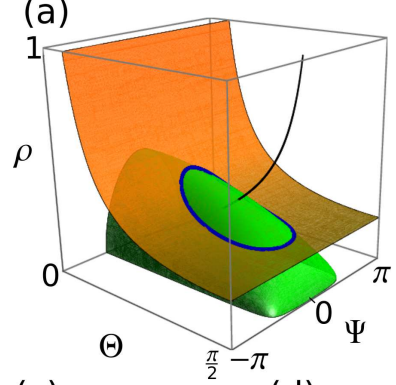

(c)

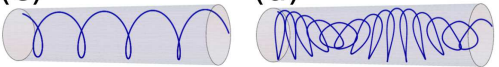

(b)

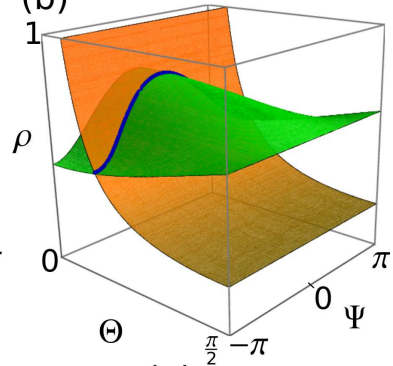

(e)

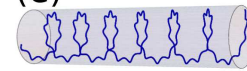

Figure 3. $\rho-\Psi-\Theta$ phase space. The intersection between $L_{z}=$ const. (orange) and $H=$ const. (green) gives the phase space trajectory. (a) helical-like swinging motion (blue intersection curve) for $L_{z}=0.2, H=1$. Black curve: fixed-point line corresponds to helical trajectories. (b) helical-like tumbling motion (blue intersection curve) for $L_{z}=0.2, H=3$. (c-e) sketch of trajectories in the channel for helical motion (c), helical-like motion (d) and tumbling motion (e).

stream for $\bar{v}_{f}<1 / \cos \Theta^{*}$, either on a left-handed helix $(\Theta>0)$ or a right-handed helix $(\Theta<0)$. Closed orbits around the fixed-point line correspond to swinging motion around a helical path [Fig. 3(d)] and open orbits [Fig. 3(b)] are complicated tumbling trajectories [Fig. 3(e)].

Now we consider hydrodynamic interactions of the microswimmer with the bounding channel wall. The flow field for neutrally buoyant swimmers in a bulk fluid is in leading order a force dipole, $\mathbf{v}(\mathbf{r})=\frac{p}{8 \pi \eta r^{2}}\left[3(\hat{\mathbf{r}} \cdot \hat{\mathbf{e}})^{2}-1\right] \hat{\mathbf{r}}$ where $\hat{\mathbf{r}}=\mathbf{r} / r$ and $\eta$ is the viscosity of the fluid. For positive dipole strength, $p>0$, the propelling apparatus of the swimmer is typically at the back (pusher), and for $p<0$ in the front (puller). Ref. [6] treated the swimmer close to a plane wall. The authors showed that hydrodynamic interactions between the swimmer and the wall lead to re-orientation due to the wall-induced vorticity $\boldsymbol{\Omega}_{W}$ and to attraction/repulsion. For example, a pusher swimming parallel to the wall is attracted and remains trapped at the wall, while a puller is repelled from the wall. We note that close to a wall the force-dipole approximation for $\boldsymbol{\Omega}_{W}$ is no longer valid. Instead, geometric details of the swimmer and thereby near fields become important for $\boldsymbol{\Omega}_{W}$. Combining flow and wall effects, the total angular velocity of a swimmer near a wall is $\boldsymbol{\Omega}(x, \Psi)=\boldsymbol{\Omega}_{W}(x, \Psi)+\boldsymbol{\Omega}_{f}(x)$. Recent work showed that hydrodynamic re-orientation is almost negligible for bacteria swimming near walls [9, 10] but rotates the swimmer at the wall. For a sufficiently weak Poiseuille flow field, stable orientations $\Psi_{W}$ for swimming at the wall exist when $\boldsymbol{\Omega}_{W}\left(R_{\mathrm{Ch}}, \Psi_{W}\right)+\boldsymbol{\Omega}_{f}\left(R_{\mathrm{Ch}}\right)=0$ [11]. Thermal fluctuations will, however, reorient the swimmer so that it leaves the wall [10].

In narrow channels a microswimmer experiences hy-

drodynamic interactions with the wall all the time. To capture the basic idea, we concentrate on 2D-trajectories and consider instead of the cylindrical channel wall, two parallel plates located at $x=1$ and $x=-1$. We calculate the wall-induced translational and angular velocities using the force-dipole approximation of Ref. [6] and obtain the equations of motion,

$$
\begin{aligned}
& \dot{x}=-\sin \Psi-\frac{3 \bar{p}\left(3 \sin ^{2} \Psi-1\right)}{64 \pi}\left(\frac{1}{(1-x)^{2}}-\frac{1}{(1+x)^{2}}\right) \\
& \dot{\Psi}=\bar{v}_{f} x-\frac{3 \bar{p} \sin \Psi \cos \Psi}{64 \pi}\left(\frac{1}{(1-x)^{3}}+\frac{1}{(1+x)^{3}}\right)
\end{aligned}
$$

where $\bar{p}=p /\left(\eta v_{0}\right)$ is the reduced dipole strength. Fig. [4 shows typical phase space plots generated from Eqs. (8) for a puller (a) and pusher (b). The swinging motion of an upstream oriented puller becomes damped and an attractive fixed point in the center exists. Repelled by both walls, the puller swims upstream along the centerline. On the other hand, a puller tumbling near the wall is attracted, on average, by the wall and stays near to it. This is indicated by the green stable trajectory. All trajectories outside the unstable red limit cycle or separatrix converge to it. However, due to thermal fluctuations the puller may cross the separatrix. A pusher behaves differently, it is attracted by the wall when oriented upstream in the center of the channel, but it is pushed away from the wall when tumbling near the wall. So, all trajectories converge towards a swinging motion about the centerline, characterized by a stable limit cycle in the $x$ - $\Psi$-plane.

To test our findings we simulate the motion of a spherical microswimmer in Poiseuille flow using the method of multi-particle collision dynamics (MPCD) [12]. It solves the Navier-Stokes equations on a coarse-grained level and calculates the flow field around the swimmer in the cylindrical microchannel taking into account both hydrodynamic interactions and thermal noise. In every simulation step randomly distributed point-particles of mass $m$ at temperature $k_{B} T$ first move ballistically for a time $\Delta t$ and then they are sorted into cubic cells of length $a$. They interact with all other particles in the cell with a specific collision rule such that momentum is conserved locally. The density of the fluid is $m N_{c} / a^{3}$ where $N_{c}$ is the average number of particles per cell. Depending on the parameters and the specific collision rule, the viscosity $\eta$ of the fluid can be calculated [12, 18].

As a model microswimmer we use a spherical squirmer of radius $R_{S}$ [13, 14]. It propels itself by a static, axisymmetric and tangential velocity field on its surface, $\mathbf{v}_{s}\left(\hat{\mathbf{r}}_{s}, \hat{\mathbf{e}}\right)=\left(B_{1}+\left(\hat{\mathbf{e}} \cdot \hat{\mathbf{r}}_{s}\right) B_{2}\right)\left[\left(\hat{\mathbf{e}} \cdot \hat{\mathbf{r}}_{s}\right) \hat{\mathbf{r}}_{\mathbf{s}}-\hat{\mathbf{e}}\right]$ where $\hat{\mathbf{r}}_{s}$ is the radial unit vector pointing from the center of the squirmer to the surface. The first mode $B_{1}$ determines the swimming speed $v_{0}=2 B_{1} / 3$ and the second mode the strength of the force dipole $p / \eta=-4 \pi B_{2} R_{S}^{2}$, so $B_{2}>0$ models a puller and $B_{2}<0$ a pusher. The squirmer has 
(a) $\operatorname{puller}(\mathrm{p}<0)$

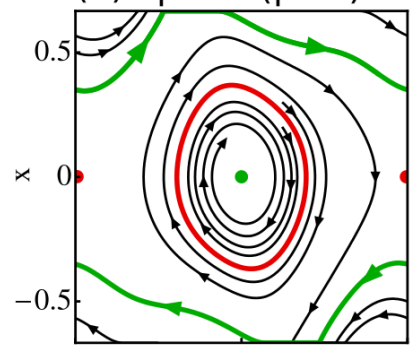

(b) pusher $(p>0)$

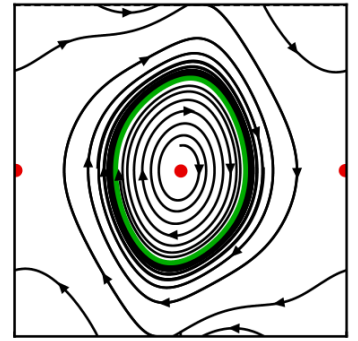

(c)

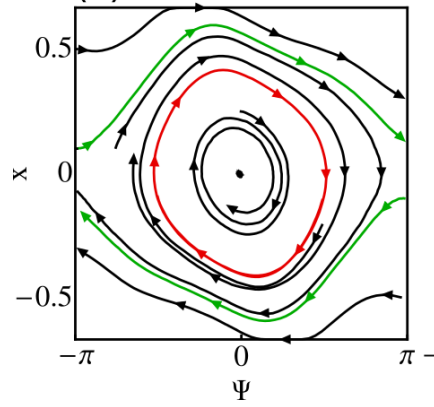

(d)

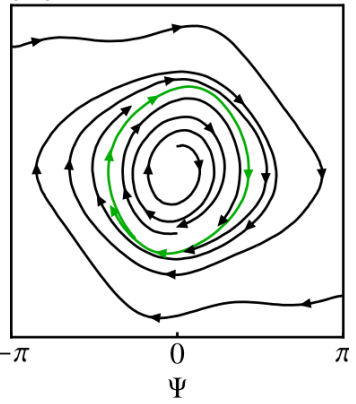

Figure 4. Phase space trajectories for a microswimmer in a narrow channel for $\bar{v}_{f}=10$ for a puller (left) and a pusher (right). Green and red indicate, respectively, stable and unstable trajectories. (a) and (b) are obtained from Eqs. (8) and (c) and (d) from MPCD simulations where we used the parameters $B_{1}=0.045, \beta=B_{2} / B_{1}= \pm 5, R_{S}=6, R_{\mathrm{Ch}}=18$, $N_{c}=30, \Delta t=0.02$ setting $a=m=k_{B} T=1$ and the collision rule MPC-AT-a [18]. Each trajectory was obtained by averaging over 10 individual runs.

already been used to investigate hydrodynamic interactions between several swimmers [14, 15] and between a swimmer and a wall [16] and has been realized in experiments quite recently [17]. To implement the squirmer in the MPCD fluid, we follow previous work [15, 19]. Figs. 4 (c)-(d) show simulated trajectories for several initial conditions for a puller (c) and a pusher (d). Our parameters are listed in the caption of Fig. 4. Although near field effects and the large extent of the swimmer play a role in the simulated dynamics, the qualitative behavior arising from hydrodynamic interactions between the swimmer and the cylindrical channel wall follows the analytical model.

In order to learn about typical relaxation times in which swimmers approach their stable trajectories, we linearize Eqs. (8) around the fixed point in the center. We obtain a harmonic oscillator equation for $\Psi$ with a friction coefficient $\gamma$ linear in the dipole strength, $\gamma=-3 p /\left(64 \pi \eta R_{\mathrm{Ch}}^{3}\right)$. For the squirmer, the estimated relaxation time for swinging motion becomes $\gamma^{-1}=$ $t_{0} \cdot 32 \bar{R}^{3} /(9 \beta)$, where $\beta=B_{2} / B_{1}, \bar{R}=R_{\mathrm{Ch}} / R_{S}$, and $t_{0}=R_{\mathrm{Ch}} / v_{0} \approx 1 \mathrm{~s}$ is a characteristic time scale for narrow microchannels. Typical values for $\beta$ range from -1 to +1 for existing microswimmers [20]. So experiments should be able to observe that microswimmers approach their

stable trajectories within seconds in sufficiently narrow channels. Similar estimates apply to the E. coli bacterium where $p \approx 0.8 p N \mu m$ was measured recently [10]. Although hydrodynamic interactions between a small microswimmer and a single wall may play no significant role, they become important in channels when the channel diameter is only few times the size of the swimmer.

In conclusion, through a formal mapping onto a Hamiltonian dynamical system we have shown that spherical microswimmers perform either an upstream oriented swinging or a tumbling motion when moving in Poiseuille flow. Hydrodynamic interactions of the swimmer with the wall stabilizes the upstream orientation of pullers in the center of the channel whereas a pusher performs stable oscillations around the centerline with a specific amplitude.

Spherical artificial swimmers with different locomotion mechanisms have been constructed and studied recently [21]. Investigating them in microfluidic channels under Poiseuille flow, the generic features presented in this article should be accessible in experiments.

We thank R. Goldstein, I. Pagonabarraga, T. Pfohl, S. Uppaluri and R. Vogel for helpful discussions and the Deutsche Forschungsgemeinschaft for financial support through the research training group GRK1558.

[1] J. A. Riffell and R. K. Zimmer, J. Exp. Biol. 210, 3644 (2007).

[2] S. Uppaluri et al. (unpublished).

[3] B. J. Nelson, I. K. Kaliakatsos, and J. J. Abbott, Annu. Rev. Biomed. Eng. 12, 55 (2010).

[4] B. ten Hagen, R. Wittkowski, and H. Löwen, Phys. Rev. E 84, 031105 (2011).

[5] J. O. Kessler, Nature (London) 313, 218 (1985).

[6] A. P. Berke, L. Turner, H. C. Berg, and E. Lauga, Phys. Rev. Lett. 101, 038102 (2008).

[7] J. Hill, O. Kalkanci, J. L. McMurry, and H. Koser, Phys. Rev. Lett. 98, 068101 (2007).

[8] R. W. Nash, R. Adhikari, J. Tailleur, and M. E. Cates, Phys. Rev. Lett. 104, 258101 (2010).

[9] G. Li and J. X. Tang, Phys. Rev. Lett. 103, 078101 (2009).

[10] K. Drescher, J. Dunkel, L. H. Cisneros, S. Ganguly, and R. E. Goldstein, Proc. Natl. Acad. Sci. USA 108, 10940 (2011).

[11] A. Zöttl and H. Stark (unpublished).

[12] A. Malevanets and R. Kapral, J. Chem. Phys. 110, 8605 (1999).

[13] J. Lighthill, Commun. Pure Appl. Math. 5, 109 (1952); J. R. Blake, J. Fluid Mech. 46, 199 (1971).

[14] T. Ishikawa, M. P. Simmonds, and T. J. Pedley, J. Fluid Mech. 568, 119 (2006).

[15] I. O. Götze and G. Gompper, Phys. Rev. E 82, 041921 (2010).

[16] I. Llopis and I. Pagonabarraga, J. Non-Newtonian. Fluid Mech. 165, 946 (2010).

[17] S. Thutupalli, R. Seemann, and S. Herminghaus, New J. 
Phys. 13, 073021 (2011).

[18] H. Noguchi, and G. Gompper, Phys. Rev. E 78, 016706 (2008).

[19] M. T. Downton and H. Stark, J. Phys. Condens. Matt. 21, 204101 (2009).

[20] A. A. Evans, T. Ishikawa, T. Yamaguchi, and E. Lauga,
Phys. Fluids 23, 111702 (2011)

[21] See, e. g., J. R. Howse et al., Phys. Rev. Lett. 99, 048102 (2007); J. Palacci et al., Phys. Rev. Lett. 105, 088304 (2010); H.-R. Jiang et al., Phys. Rev. Lett. 105, 268302 (2010). 